\title{
Kidney Cancer Research Network of Canada (KCRNC) consensus statement on the role of renal mass biopsy in the management of kidney cancer
}

Luke T. Lavallée, MDCM, MSc, FRCSC ${ }^{1,2}$; Kristen McAlpine, MD ${ }^{1}$; Anil Kapoor, MD, FRCSC $^{3}$; Frédéric Pouliot, MD, PhD, FRCSC ${ }^{4}$; Ross Mason, MD, MSc, FRCSC ${ }^{5}$; Philippe Violette, MD, FRCSC ${ }^{6}$; Rahul K. Bansal, MD, MCh, FRCSC ${ }^{7}$; Patrick Richard, MD, MSc, FRCSC $^{8}$; Pierre I. Karakiewicz, MD, MPH, FRCSC ${ }^{9}$; Bimal Bhindi, MD, CM, MSc, FRCSC ${ }^{10}$; Ranjena Maloni ${ }^{11}$; Stephen Pautler, MD, FRCSC ${ }^{12}$; Jean-Baptiste Lattouf, MD, FRCSC ${ }^{9}$; Wassim Kassouf, MD, CM, FRCSC ${ }^{13}$; Simon Tanguay, MD, FRCSC ${ }^{13}$; Alan So, MD, FRCSC ${ }^{14}$; Ricardo A. Rendon, MD, MSc, FRCSC ${ }^{5}$; Rodney H. Breau, MD, MSc, FRCSC ${ }^{1,2}$

${ }^{1}$ Division of Urology, University of Ottawa, Ottawa, ON, Canada; ${ }^{2}$ Ottawa Hospital Research Institute, University of Ottawa, Ottawa, ON, Canada; ${ }^{3}$ Departments of Surgery (Urology) and Oncology, McMaster University, Hamilton, ON, Canada; ${ }^{4}$ Department of Surgery, Division of Urology, Université Laval, Quebec City, QC, Canada; ${ }^{5}$ Department of Urology, Dalhousie University, Halifax, NS, Canada; ${ }^{6}$ Departments of Health Research Methods Evidence and Impact and Surgery, McMaster University, Hamilton, ON, Canada; ${ }^{7}$ Department of Surgery, University of Manitoba, Winnipeg, MB, Canada; ${ }^{8}$ Division of Urology, University of Sherbrooke, Sherbrooke, QC, Canada; ${ }^{9}$ Department of Surgery, University of Montreal, Montreal, QC, Canada; ${ }^{10}$ Department of Surgery, Section of Urology, University of Calgary, Calgary, AB, Canada; ${ }^{11}$ Kidney Cancer Research Network of Canada;

${ }^{12}$ Department of Surgery, Division of Urology, Western University, London, ON, Canada; ${ }^{13}$ Department of Surgery, Division of Urology, McGill University, Montreal, QC, Canada; ${ }^{14}$ Department of Urologic Sciences, University of British Columbia, Vancouver, BC, Canada

Cite as: Can Urol Assoc J 2019 September 17; Epub ahead of print. http://dx.doi.org/10.5489/cuaj.6176

Published online September 17, 2019

$* * *$

\section{Introduction}

The pervasive use of diagnostic imaging has led to an increase in the incidental detection of small renal masses. ${ }^{1-4}$ The assessment and management of a patient with a renal mass should vary based on mass characteristics and on the individual patient's health and personal preferences.

Renal mass biopsy is a diagnostic test used to obtain tissue from a suspicious mass in the kidney. Several patient factors and mass characteristics should be considered to determine when a biopsy is a useful test for a patient. Recently, there have been a number of published series on renal mass biopsy that discuss which patient populations benefit from this diagnostic test. ${ }^{5-7}$

The objectives of this consensus statement are: 1) to review and synthesize the evidence on renal mass biopsy; and 2) to highlight important concepts and provide guidance regarding the role of renal mass biopsy. The statements contained in this report were based on the best available evidence and developed by expert consensus. It is expected that these statements will 
be used to guide care in Canada and that some variability in practice will exist for individual patients and regional practice variation.

The scientific literature available for this consensus statement was of low-to-moderatequality. The evidence reported on renal mass biopsy is predominantly comprised of retrospective cohort series of patients managed at high-volume centers. ${ }^{5-7}$ Recently, a systematic review and meta-analysis of renal mass biopsy was published, which summarizes the best available evidence on the diagnostic ability and safety of this test. ${ }^{7}$

\section{Management options for renal masses}

Several factors should be considered during consultation of a patient referred with a renal mass. Individual patient considerations include age, sex, comorbidities, medications, and performance status, as these factors are associated with the probability of renal malignancy, the probability of biopsy/treatment-related adverse events, and/or the patient's overall life expectancy. ${ }^{8}$ Mass characteristics that should be considered include size, location, appearance on imaging, number of masses, and presence of cystic components, as these factors are associated with probability of malignancy and the diagnostic performance of biopsy. Most importantly, patient preferences and values must be considered to facilitate shared decision-making about diagnostic tests and management.

Small renal masses are lesions in the kidney that are typically defined as $<4 \mathrm{~cm}$ in diameter. Although the majority of these masses are malignant, many malignant kidney masses do not harbor aggressive histology (low-grade, low risk of progression), and approximately 20\% are benign. ${ }^{8-10}$ Common management options include surgical removal (partial or radical nephrectomy), thermal ablation, active surveillance, and watchful waiting. ${ }^{11-14}$ The choice of management should be tailored to the patient based on medical factors, mass characteristics, and the patient's values and preferences.

Renal masses $>4 \mathrm{~cm}$ have a higher probability of being malignant. ${ }^{10,15,16}$ The options for management are fewer compared to small masses, and most patients with non-metastatic disease who are suitable for intervention are counselled to proceed with surgical removal of the mass. Biopsy of a localized large $(>4 \mathrm{~cm})$ renal mass is not routinely recommended unless there is clinical suspicion of a non-renal cell carcinoma (RCC) mass or abscess. ${ }^{5,16}$

\section{Renal mass biopsy to guide clinical decision-making}

1. Renal mass biopsy should be offered to patients with a renal mass when the result of the biopsy will alter their management.

Renal mass biopsy is a diagnostic test. Therefore, like any diagnostic test, it should be performed if the result will influence management. Historically, almost all medically fit patients with a solid enhancing renal mass suspicious for RCC were recommended for surgical treatment to avoid any risk of metastatic spread. It is now known that $20 \%$ of small renal masses are benign and most of the malignant masses have low metastatic potential. ${ }^{9,10,17}$ In recent years, 
greater understanding of the natural history of small renal masses has led to increased use of active surveillance to avoid complications of treatment. ${ }^{18,19}$ While surgery for a small renal mass is usually curative, based on a recent systematic review and meta-analysis, complications of surgery occur in $21 \%$ of patients, with $7 \%$ of patients having a severe (Clavien grade 3-5) complication. ${ }^{20} \mathrm{~A}$ diagnostic test, such as renal mass biopsy, that may safely allow some patients with benign masses to avoid treatment, is useful. Current rates of renal mass biopsy in Canada are not known. ${ }^{21}$ Many studies report rates of biopsy in patients who received treatment (e.g., surgery), however, these do not account for patients who may have received biopsy and avoided surgery. A Canadian study reported that at centers where renal mass biopsy is more frequently performed for patients with small renal masses, there are fewer benign masses surgically excised. $^{22}$ In this study, at centers where the renal mass biopsy rate was $63 \%$, the rate of benign pathology for surgically excised masses was $5 \% .{ }^{22}$ Comparatively, centers with a biopsy rate of $12 \%$ had an $11 \%$ benign pathology rate for surgically excised masses. ${ }^{22}$ It is, however, unknown if any of the renal masses spared from surgery after biopsy progressed and caused symptoms or were false negative biopsies. Also, at centers that have higher rates of renal mass biopsy for small renal masses, more patients were submitted to the risks of biopsy. ${ }^{22}$ Because both upfront treatment of small renal masses and renal mass biopsy expose patients to risks, it is important to consider in which patients renal mass biopsy will influence management.

\section{Renal mass biopsy as a diagnostic test}

2. Renal mass biopsy should include at least 2-3 core biopsies to sample the mass. Fine-needle aspiration is not sufficient.

3. The diagnostic accuracy of renal mass biopsy varies by hospital, mass size, mass location, and patient factors.

The utility of renal mass biopsy depends on its ability to provide a diagnosis of malignant vs. benign histology. Importantly, renal mass biopsy should include multiple core tissue samples (at least 2-3) using a large bore needle (16-18 gauge) through a coaxial sheath. ${ }^{12}$ Core needle biopsies are superior in diagnostic ability when compared to fine-needle aspiration of a mass and should be considered standard of care for renal mass biopsy. ${ }^{23}$

Numerous series of renal mass biopsy for small renal masses performed at experienced centers have been published, including several with Canadian data. ${ }^{5-7}$ A recent systematic review and meta-analysis including 57 studies and 5228 patients reported on the diagnostic characteristics of renal mass biopsy. The overall diagnostic rate was $92 \%$ (interquartile range [IQR] 81-97\%) and the non-diagnostic biopsy rate ranged from $0-23 \%{ }^{7}$ Diagnostic rate indicates the percentage of renal mass biopsies for which the pathologist was able to provide a diagnosis of malignant or benign pathology based on the tissue sampled. A non-diagnostic biopsy indicates that only normal renal tissue was sampled (i.e., the biopsy missed the mass) or the pathologist was unable to differentiate benign vs. malignant pathology of the mass. ${ }^{24}$ A true positive biopsy result indicates that the biopsy pathology was concordant with the surgical 
pathology (e.g., both showed RCC). A true negative biopsy would indicate that there is no malignancy in the mass if the biopsy is benign. Since most series do not remove masses with benign findings on biopsy, the true negative rate is frequently unknown, and calculation of sensitivity and specificity is limited. The largest Canadian series on renal mass biopsy recently reported a diagnostic rate of $90 \%(n=476)$ for patients with a small renal mass. ${ }^{6}$ In this series, for patients with a non-diagnostic first renal mass biopsy who then underwent a second biopsy $(\mathrm{n}=24), 83 \%$ had a diagnostic second renal mass biopsy. ${ }^{6}$ The true positive rate for renal mass biopsy has been reported between $74 \%$ and $100 \% .{ }^{7}$ However, the concordance between tumor (mass) grade on biopsy and grade on surgical pathology is 62.5\% (IQR 52.1-72.1\%). ${ }^{7}$

A team of physicians with experience performing and analyzing the results of renal mass biopsy is important. Centers with an experienced radiologist to perform renal mass biopsy and a genitourinary pathologist to review the tissue have reported consistently high diagnostic rates. ${ }^{5,725,26}$ It is not known if these results can be replicated in lower-volume centers. Systematic reviews have shown variability in diagnostic accuracy of renal mass biopsy does exist between centers, therefore, individual centers are encouraged to review their institutional experience when possible. ${ }^{7,27}$ Patient factors and mass characteristics may alter the difficulty and decrease the accuracy of renal mass biopsy. Smaller mass diameter, cystic components, and longer skinto-mass distance reduce the diagnostic yield of a renal mass biopsy.,28

\section{Safety of renal mass biopsy}

4. Renal mass biopsy is safe, with low rates of complications when performed at experienced centers in properly selected patients. Patients should be informed of the risk of complications.

The benefit-to-risk ratio of a diagnostic test should be considered prior to ordering the test. This is especially true for invasive tests, including renal mass biopsy. The overall risk of complications following renal mass biopsy in published series is $8 \%$, with the majority of these being Clavien 1 complications. ${ }^{7}$ The most common risk of renal mass biopsy is bleeding, which is usually minor and limited to a self-resolving perirenal hematoma (4.3\%). ${ }^{7}$ Mild hematuria and back pain are reported in $3.2 \%$ and $3 \%$ of patients, respectively. ${ }^{7}$ Significant bleeding requiring blood transfusion was reported in $0.7 \%$ of patients. ${ }^{7}$ Clavien $\geq 2$ complications are uncommon $(<0.5 \%)$ in reported series. ${ }^{7}$ The risk of complications varies by center, patient, and mass characteristics, and these should be considered when counselling patients.

Tumor seeding of the biopsy tract may be a concern when a malignant mass is sampled. Very few cases of tumor seeding along the biopsy tract after renal mass biopsy have been reported in contemporary series. ${ }^{7,12}$ One recent case series from a referral center in the U.K. reported evidence of RCC along the biopsy tract of seven patients based on examination of the surgical specimen. ${ }^{29}$ Tumor seeding following renal mass biopsy causing clinical manifestations is currently felt to be a low risk to patients. 
Anticoagulation and antiplatelet medications should be stopped if safe to do so prior to renal mass biopsy to reduce the risk of bleeding complications. ${ }^{30}$ For high-risk patients (e.g., recent coronary artery stenting, recent venous thromboembolism, high CHADS score) consultation with a thrombosis expert is recommended. Thrombosis Canada has a useful online tool to aid physicians when determining the optimal timing to stop and restart anticoagulants and antiplatelets around procedures, including renal mass biopsies (https://thrombosiscanada.ca/guides/). ${ }^{31}$ Additional guidance for management of anticoagulation and antiplatelets around the time of renal mass biopsy can also be found in the Canadian Urological Association (CUA) guideline on perioperative thromboprophylaxis. ${ }^{32}$

\section{Predictive tools for patient with renal masses}

The risk that a renal mass is malignant is associated with patient factors and mass characteristics. A number of clinical tools have been created to assist physicians and patients in the decisionmaking process by attempting to predict the chance a renal mass is malignant. Nomograms require the input of patient and mass characteristics and can provide a percentage chance that a mass is cancerous. ${ }^{8,33}$ One nomogram studied used patient demographic factors and the R.E.N.A.L nephrometry score to predict whether a mass was benign or malignant, as well as if it was high-grade or low-grade. ${ }^{33}$ This nomogram was able to predict malignancy (area under the curve [AUC] 0.76) and the grade of the mass (AUC 0.73) with good accuracy but has not been externally validated. ${ }^{33}$ Classification trees have also been created to guide physician decisionmaking when assessing a patient with a small renal mass. These clinical tools are based on patient factors and mass characteristics and are meant to follow a physician's thought process. Recently, a Canadian-based classification tree for small renal masses was externally validated and updated, with an accuracy of $87 \%$ (95\% confidence interval $0.84-0.89$ ) at predicting for malignant pathology on renal mass biopsy. ${ }^{34}$

Use of predictive tools to determine an individual patient's pre-test probability of a malignant mass (in this case pre-renal mass biopsy) contributes to personalized care, and may assist in determining if a biopsy is required. Despite the availability of these predictive tools, the ability to differentiate between high-grade and low-grade histology using currently available tools is limited, and care must be taken when using a predictive tool to determine if a biopsy should be performed. ${ }^{35}$

\section{Renal mass biopsy for small renal masses}

5. Renal mass biopsy should routinely be discussed with patients with a small renal mass prior to management.

6. Shared decision-making should be used to determine if renal mass biopsy will be performed. Patients should be informed of the possible benefits and harms, what is known about the diagnostic accuracy of the biopsy, and how the biopsy should be 
interpreted. Patients' values and preferences should be elicited. Most importantly, it should be determined whether the results of the biopsy will influence management.

7. Patients who have a non-diagnostic renal mass biopsy for a small renal mass should be counselled on the benefits and harms of a repeat biopsy.

8. Patients who have a renal mass biopsy with benign histology should be informed about the risk of a false negative biopsy and should be monitored.

Renal mass biopsy can be an important diagnostic tool to guide the management of a patient with a renal mass. Prior to ordering a renal mass biopsy, a physician must evaluate the patient's values and preferences with respect to management of the renal mass. While renal mass biopsy is usually well-tolerated, it is an invasive procedure associated with risks. Patients must be counselled on the rationale for a renal mass biopsy, how the results (malignant, nonmalignant, non-diagnostic) may alter their management choices, the side effects of a biopsy, and alternatives management options. Patient counselling should encourage shared decision-making and a patient-centered approach to care.

A renal mass biopsy provides three possible histological results; malignant, benign, or non-diagnostic. When a renal mass biopsy is malignant, the physician should discuss management options with the patient. When a renal mass biopsy is reported benign, patients should be monitored with imaging to ensure there are no concerning interval changes in the size or appearance of the mass. The diagnostic accuracy of renal mass biopsy at experienced centers is good, however, there is still the possibility of a false negative test result (i.e., benign biopsy reported when a malignancy is present). The false negative rate of renal mass biopsies in one Canadian series was 3.5\%; however, most renal mass biopsy series do not report the false negative rate, as masses with a benign biopsy are not removed. Therefore, a range of false negative rates may be expected based on center experience and patient selection. ${ }^{5,36}$ One example of a particularly challenging diagnosis is differentiating oncocytoma and chromophobe RCC. A reasonable approach to monitoring after a benign renal mass biopsy would include imaging with an ultrasound or computed tomography (CT) scan at six months and 12 months after the renal mass biopsy. The followup imaging schedule can then be adapted based on patient factors and mass characteristics, such as the patient age, mass size, and growth pattern after the first year.

For patients with a non-diagnostic renal mass biopsy, management may include monitoring, repeat biopsy attempt, or proceeding directly to definitive treatment. After a nondiagnostic renal mass biopsy, patients should be counselled on the benefits and harms of a repeat biopsy. If it is felt the results of repeat biopsy may alter management, repeat biopsy may be offered.

Patients in whom renal mass biopsy should not be recommended

9. Renal mass biopsy should not be recommended to patients in whom active surveillance or watchful waiting will be recommended irrespective of the biopsy result because of competing risks. 
10. Renal mass biopsy should not be recommended to patients who will want to proceed with definitive management irrespective of the biopsy result.

11. Renal mass biopsy should not be performed in patients with a renal mass showing classic radiological appearance of an angiomyolipoma.

Renal mass biopsy should be offered to patients when the biopsy result may alter the management approach they select. For some patients, the results of a biopsy, malignant or benign, are unlikely to alter the management options they select. For very elderly, highly comorbid, or frail patients, the competing risks of mortality from other causes outweigh the risk of death from small renal mass even if a biopsy reveals RCC. In these patients, whom active surveillance or watchful waiting will be recommended irrespective of the biopsy outcome, renal mass biopsy should not be performed.

For healthy patients with a long life expectancy and a low risk of significant morbidity from definitive treatment, it is important to discuss the role of renal mass biopsy and how the results may impact their treatment choices. Some patients will prefer definitive management because they are unwilling to accept any uncertainty after a renal mass biopsy or because they want to avoid a long period of imaging surveillance. In these patients, proceeding with definitive treatment is recommended and a renal mass biopsy should not be performed.

Angiomyolipomas (AMLs) are benign renal masses that contain fat, smooth muscle, and blood vessels. ${ }^{37}$ The majority of these lesions contain abundant amounts of fat visible on imaging, making the diagnosis of AML on cross-sectional imaging reliable. ${ }^{38}$ Fat-containing RCCs are rare. Renal masses with classic radiological features of an AML do not require a renal mass biopsy to confirm the diagnosis.

\section{Renal mass biopsy of cystic renal masses}

12. Biopsy of cystic renal masses may be considered if there is a significant solid component amenable to biopsy. Renal masses without a solid component should not be biopsied due to low diagnostic yield.

The use of renal mass biopsy for cystic and solid renal masses is different. First, the diagnostic yield is lower for cystic tumors, given the large fluid-filled area of the mass. ${ }^{7,12}$ Second, the risk of puncture and spillage of the cystic fluid is a concern. ${ }^{12}$ Finally, in comparison to matched solid-enhancing renal masses, renal masses with a large cystic component are associated with a less aggressive natural history and a lower risk of metastases. ${ }^{39,40}$ Therefore, unless there is a solid, nodular, enhancing component in the cystic renal mass, these masses should not routinely be biopsied.

\section{Other indications for renal mass biopsy}

13. Renal mass biopsy should be performed when a pathology other than RCC is suspected for a mass that may require management (e.g., lymphoma, metastatic lesion). 


\section{Renal mass biopsy or biopsy of a metastatic lesion should be considered to obtain a diagnosis in patients with suspected metastatic RCC.}

15. Renal mass biopsy should be performed prior to, or at the time of, thermal ablation or radiotherapy of the mass.

For patients with renal masses that do not have an imaging phenotype typical of RCC or who have a past history of disease that may cause a non-RCC renal mass, a renal mass biopsy should be considered. Other processes can cause renal masses on imaging, including upper tract urothelial cell carcinoma, infection, inflammation, lymphoproliferative disorders, and metastatic cancer from a different primary. Eliciting a history of symptoms or a past medical history fitting these other causes is important in the assessment of a patient referred with a renal mass. Patients with renal masses that may be suspicious for an upper tract urothelial cell carcinoma should have urine cytology and possible endoscopic evaluation, depending on the estimated probability of a collecting system malignancy. Patients with inflammatory or infectious causes of a renal mass may have systemic symptoms of fever, chills, rash, or imaging with significant perinephric stranding. Lymphoma is rarely the cause of a solitary renal mass, however, it is the most common hematological malignancy to appear in a kidney. ${ }^{41}$ Renal involvement of lymphoma can vary in appearance from multifocal, ill-defined, hypovascular lesions of different sizes to an infiltrative mass extending from the retroperitoneum with or without lymphadenopathy. ${ }^{42}$ Finally, patients with a history of a concurrent or prior non-RCC malignancy must also be evaluated for the possibility of a metastatic deposit. Solitary metastatic lesions are rarely present in the kidney, but have been reported from melanoma, lung cancer, colon cancer, and thyroid cancer. ${ }^{41}$ When there is a concern that a renal mass could represent a pathology other than RCC, a renal mass biopsy should be considered.

Renal mass biopsy should be considered in patients with de novo metastatic disease suspected to be RCC in origin. A biopsy of the renal mass or a metastatic deposit are both reasonable options to obtain a tissue diagnosis if upfront systemic therapy is contemplated. A renal mass biopsy of the kidney provides the most reliable pathology in many cases. ${ }^{43,44}$ Confirming the diagnosis and histology of the renal mass is important, as it may impact the options for systemic therapy and eligibility for clinical trials.

Finally, for patients in whom radiofrequency ablation, cryoablation, or radiotherapy of the renal mass is being considered, a renal mass biopsy should be performed before or at the time of the treatment, depending on the patient's clinical status and ability to tolerate multiple procedures. ${ }^{12,14}$ If the patient was to develop metastatic disease following treatment, the tissue obtained in the renal mass biopsy could be used to guide systemic treatment. Additionally, benign pathology may help avoid overtreatment. When possible, a renal mass biopsy should be performed prior to ablation or radiotherapy to allow better patient counselling. In high-risk patients (e.g., on anticoagulation) who are unable or unwilling to receive two procedures (biopsy and treatment), a biopsy should be performed at the time of the treatment. 


\section{Summary}

Renal mass biopsy is an effective and safe diagnostic tool for properly selected patients. As a guiding principle, renal mass biopsy should be reserved for patients in whom the results will affect their management choices. Shared decision-making between physicians and patients should be used when considering this test to investigate a renal mass. 


\section{References}

1. Turner R, Morgan T, Jacobs BL. Epidemiology of the small renal mass and the treatment disconnect phenomenon. Urol Clin North Am 2018;44:147-54. https://doi.org/10.1016/j.ucl.2016.12.001

2. Siegel RL, Miller KD, Jemal A. Cancer Statistics, 2018. CA Cancer J Clin 2018;67:7-30. https://doi.org/10.3322/caac.21387

3. Kane CJ, Mallin K, Ritchey J, et al. Renal cell cancer stage migration. Cancer 2008;113:78-83. https://doi.org/10.1002/cncr.23518

4. De P, Otterstatter MC, Marrett LD, et al. Trends in incidence, mortality, and survival for kidney cancer in Canada, 1986-2007. Cancer Causes Control 2014;25:1271-81. https://doi.org/10.1007/s10552-014-0427-x

5. Leveridge MJ, Finelli A, Kachura JR, et al. Outcomes of small renal mass needle core biopsy, non-diagnostic percutaneous biopsy, and the role of repeat biopsy. Euro Urol 2011;60:578-84. https://doi.org/10.1016/j.eururo.2011.06.021

6. Richard PO, Jewett MAS, Bhatt JR, et al. Renal tumor biopsy for small renal masses: A single-center 13-year experience. Eur Urol 2015;68:1007-13. https://doi.org/10.1016/j.eururo.2015.04.004

7. Marconi L, Dabestani S, Lam TB, et al. Systematic review and meta-analysis of diagnostic accuracy of percutaneous renal tumor biopsy. Eur Urol 2016;69:660-73. https://doi.org/10.1016/j.eururo.2015.07.072

8. Lane B, Babineau D, Kattan M, et al. A preoperative prognostic nomogram for solid enhancing renal tumors $7 \mathrm{~cm}$ or less amenable to partial nephrectomy. J Urol 2007;:42934. https://doi.org/10.1016/j.juro.2007.03.106

9. Pahernik S, Ziegler S, Roos F, et al. Small renal tumors: Correlation of clinical and pathological features with tumor size. J Urol 2007;178:414-7. https://doi.org/10.1016/j.juro.2007.03.129

10. Bhindi B, Thompson RH, Lohse CM, et al. The probability of aggressive vs. indolent histology based on renal tumor size: Implications for surveillance and treatment. Eur Urol 2018;74:489-97. https://doi.org/10.1016/j.eururo.2018.06.003

11. Novick AC, Campbell SC, Belldegrun A, et al. Guideline for management of the clinical stage T1 renal mass. J Urol 2009;182:1271-9. https://doi.org/10.1016/j.juro.2009.07.004

12. Campbell S, Uzzo RG, Allaf ME, et al. Renal mass and localized renal cancer: AUA guideline. J Urol 2017;198:520-9. https://doi.org/10.1016/j.juro.2017.04.100

13. Ljungberg B, Albiges L, Bensalah K, et al. EAU guidelines on renal cell carcinoma 2018. Euro Urol 2018;29:451-8.

14. Jewett M, Rendon R, Lacombe L, et al. Canadian guidelines for the management of small renal masses (SRM). Can Urol Assoc J 2015;99:160-3. https://doi.org/10.5489/cuaj.2969

15. Thompson R, Kurta J, Kaag M, et al. Tumor size is associated with malignant potential in renal cell carcinoma cases. J Uro 2009;181: 2033-6.

https://doi.org/10.1016/j.juro.2009.01.027 
16. Rendon R, Kapoor A, Breau R, et al. Surgical management of renal cell carcinoma: Canadian Kidney Cancer Forum Consensus. Can Urol Assoc J 2014;8:E398-412. https://doi.org/10.5489/cuaj.1894

17. Lane BR, Demirjian S, Derweesh IH, et al. Survival and functional stability in chronic kidney disease due to surgical removal of nephrons: Importance of the new baseline glomerular filtration rate. Eur Urol 2015;68:996-1003.

https://doi.org/10.1016/j.eururo.2015.04.043

18. Jewett MAS, Mattar K, Basiuk J, et al. Active surveillance of small renal masses: Progression patterns of early stage kidney cancer. Eur Urol 2011;60:39-44. https://doi.org/10.1016/j.eururo.2011.03.030

19. Pierorazio PM, Johnson MH, Ball MW, et al. Five-year analysis of a multi-institutional prospective clinical trial of delayed intervention and surveillance for small renal masses: The DISSRM registry. Eur Urol 2015;68:408-15. https://doi.org/10.1016/j.eururo.2015.02.001

20. Cacciamani G, Medina L, Gill T, et al. Impact of surgical factors on robotic partial nephrectomy outcomes: Comprehensive systematic review and meta-analysis. $J$ Urol 2018;200:258-74. https://doi.org/10.1016/j.juro.2017.12.086

21. Richard PO, Martin L, Lavallée LT, et al. Identifying the use and barriers to the adoption of renal tumour biopsy in the management of small renal masses. Can Urol Assoc $J$ 2018;12:260-6. https://doi.org/10.5489/cuaj.5065

22. Richard PO, Lavallée LT, Pouliot F, et al. Is routine renal tumor biopsy associated with lower rates of benign histology following nephrectomy for small renal masses? J Urol 2018;4:731-6. https://doi.org/10.1016/j.juro.2018.04.015

23. Patel HD, Johnson MH, Pierorazio PM, et al. Diagnostic accuracy and risks of biopsy in the diagnosis of a renal mass suspicious for localized renal cell carcinoma: Systematic review of the literature. J Urol 2017;195:1340-7. https://doi.org/10.1016/j.juro.2015.11.029

24. Tomaszewski JJ, Uzzo RG, Smaldone MC. Heterogeneity and renal mass biopsy: A review of its role and reliability rationale for expanded RMB indications. Cancer Biol Med 2014;11:162-72.

25. Richard PO, Jewett MAS, Tanguay S, et al. Safety, reliability, and accuracy of small renal tumour biopsies: Results from a multi-institution registry. BJU Int 2017;119:543-9. https://doi.org/10.1111/bju.13630

26. Kriegshauser JS, Patel MD, Young SW, et al. Factors contributing to the success of ultrasound-guided native renal biopsy. J Ultrasound Med 2016;35:381-7. https://doi.org/10.7863/ultra.15.05023

27. Patel HD, Johnson MH, Pierorazio PM, et al. Diagnostic accuracy and risks of biopsy in the diagnosis of a renal mass suspicious for localized renal cell carcinoma: Systematic review of the literature. J Urol 2016;195:1340-7. https://doi.org/10.1016/j.juro.2015.11.029 
28. Prince J, Bultman E, Hinshaw L, et al. Patient and tumor characteristics can predict nondiagnostic renal mass biopsy findings. J Urol 2015;193:1899-1904. https://doi.org/10.1016/j.juro.2014.12.021

29. Macklin PS, Sullivan ME, Tapping CR, et al. Tumor seeding in the tract of percutaneous renal tumor biopsy: A report on seven cases from a UK tertiary referral centre. Eur Urol 2019;75:861-7. https://doi.org/10.1016/j.eururo.2018.12.011

30. Brachemi S, Bollee G. Renal biopsy practice: What is the gold standard? World $J$ Nephrol 2014;3:287-94. https://doi.org/10.5527/wjn.v3.i4.287

31. Thrombosis Canada. Available at: https://thrombosiscanada.ca/guides/\#. Published 2019. Accessed April 10, 2019.

32. Violette PD, Lavallée LT, Kassouf W, et al. Canadian Urological Association guideline on perioperative thromboprophylaxis and management of anticoagulation. Can Urol Assoc J 201913:105-14. https://doi.org/10.5489/cuaj.5828

33. Kutikov A, Smaldone C, Egleston B, et al. Anatomic features of enhancing renal masses predict malignant and high-grade pathology: A preoperative nomogram using the RENAL nephrometry score. Eur Urol 2011;60:241-8. https://doi.org/10.1016/j.eururo.2011.03.029

34. Organ M, MacDonald L, Jewett M, et al. Classification tree for the prediction of malignant disease and the prediction of non-diagnostic biopsies in patients with small renal masses. Can Urol Assoc J 2019;13:115-9. https://doi.org/10.5489/cuaj.5196

35. Jeldres C, Sun M, Liberman D, et al. Can renal mass biopsy assessment of tumor grade be safely substituted for by a predictive model? JURO 2009;182:2585-9. https://doi.org/10.1016/j.juro.2009.08.053

36. Schmidbauer J, Remzi M, Memarsadeghi M, et al. Diagnostic accuracy of computed tomography-guided percutaneous biopsy of renal masses. Eur Urol 2008;53:1003-12. https://doi.org/10.1016/j.eururo.2007.11.041

37. Flum AS, Hamoui N, Said MA, et al. Update on the diagnosis and management of renal angiomyolipoma. J Urol 2016;195:834-46. https://doi.org/10.1016/j.juro.2015.07.126

38. Dyer R, DiSantis DJ, McClennan BL, et al. Simplified imaging approach for evaluation of the solid renal mass in adults. Radiology 2008;247:331-43. https://doi.org/10.1148/radiol.2472061846

39. Chandrasekar T, Ahmad A, Fadaak K, et al. Natural history of complex renal cysts: Clinical evidence supporting active surveillance. J Urol 2018;199:633-40. https://doi.org/10.1016/j.juro.2017.09.078

40. Bhatt JR, Jewett MAS, Richard PO, et al. Multilocular cystic renal cell carcinoma: Pathological T staging makes no difference to favorable outcomes and should be reclassified. J Urol 2016;196:1350-5. https://doi.org/10.1016/j.juro.2016.05.118

41. Huang H, Tamboli P, Karam JA. Secondary malignancies diagnosed using kidney needle core biopsies: A clinical and pathological. Hum Pathol 2016;52:55-60. https://doi.org/10.1016/j.humpath.2015.12.028 
42. Sheth S, Ali S, Fishman E. Imaging of renal lymphoma: Patterns of disease with pathologic correlation. RadioGraphics 2006;26:1151-69. https://doi.org/10.1148/rg.264055125

43. Abel J, Culp S, Matin S, et al. Percutaneous biopsy of primary tumor in metastatic renal cell carcinoma to predict high-risk pathologic features: Comparison with nephrectomy assessment. J Urol 2016;184:1877-81. https://doi.org/10.1016/j.juro.2010.06.105

44. Abel EJ, Carrasco A, Culp SH, et al. Limitations of preoperative biopsy in patients with metastatic renal cell carcinoma: Comparison to surgical pathology in 405 cases. BJU Int 2012;110:1742-6. https://doi.org/10.1111/j.1464-410X.2012.11124.X

COMPETING INTERESTS: Dr. Lavallée has been an advisory board member for Ferring and Sanofi; and received a grant from Sanofi. Dr. Kapoor has attended advisory boards for and participated in clinical trials supported by Amgen, Astellas, Janssen, GSK, Novartis, Pfizer, and Sanofi. Dr. Pouliot has been an advisory board member for Amgen, Astellas, and Pfizer; has been a speaker for Sanofi; and has received payment/grants/honoraria from Amgen, Astellas, AstraZeneca, Janssen, Pfizer, and Sanofi. Dr. Violette has been a speaker for Janssen and Sanofi (with no honoraria). Dr. Richard has been an advisory board member for BMS and Sanofi; has been a speaker for Abbvie, Amgen, Astellas, Ferring and Janssen; and has participated in clinical trials for Calithera and Lidds Pharma. Dr. Karakiewicz has been an advisory board member for Pfizer; has received payment for advisory board presentations from Abbvie, Astellas, Ferring, Janssen, and Pfizer; and has received a research grant from Pfizer. Dr. Lattouf has been an advisory board member for and received honoraria from Abbvie, AstraZeneca, Bayer, Novartis, Pfizer, and Takeda. Dr. Kassouf has received grants/ honoraria from Astellas, AstraZeneca, Janssen, Merck, and Roche. Dr. Tanguay has been an advisory board member for Pfizer; and received a travel grant from Sanofi. Dr. So has been a speaker for Amgen, Astellas, and Janssen. Dr. Rendon has been an advisory board member and speaker for and received honoraria from Abbvie, Amgen, Astellas, Astra Zeneca, Bayer, Ferring, Jansen, and Sanofi. The remaining authors report no competing personal or financial interests related to this work. 


\section{Summary of consensus statements}

1. Renal mass biopsy should be offered to patients with a renal mass when the result of the biopsy may alter their management.

2. Renal mass biopsy should include at least 2-3 core biopsies to sample the mass. Fine needle aspiration is not sufficient.

3. The diagnostic accuracy of renal mass biopsy varies by hospital, mass size, mass location, and patient factors.

4. Renal mass biopsy is safe with low rates of complications when performed at experienced centres in properly selected patients. Patients should be informed of the risk of complications.

5. Renal mass biopsy should routinely be discussed with patients with a small renal mass prior to management.

6. Shared decision-making should be used to determine if renal mass biopsy will be performed. Patients should be informed of the possible benefits and harms, what is known about the diagnostic accuracy of the biopsy, and how the biopsy should be interpreted. Patients' values and preferences should be elicited. Most importantly, it should be determined whether the results of the biopsy will influence management.

7. Patients who have a non-diagnostic renal mass biopsy for a small renal mass, should be counseled on the benefits and harms of a repeat biopsy.

8. Patients who have a renal mass biopsy with benign histology, should be informed about the risk of a false negative biopsy and should be monitored.

9. Renal mass biopsy should not be recommended to patients in whom active surveillance or watchful waiting will be recommended irrespective of the biopsy result because of competing risks.

10. Renal mass biopsy should not be recommended to patients who will want to proceed with definitive management irrespective of the biopsy result.

11. Renal mass biopsy should not be performed in patients with a renal mass showing classic radiologic appearance of an angiomyolipoma.

12. Biopsy of cystic renal masses may be considered if there is a significant solid component amenable to biopsy. Renal masses without a solid component should not be biopsied due to low diagnostic yield.

13. Renal mass biopsy should be performed when a pathology other than renal cell carcinoma is suspected for a mass that may require management (ex: lymphoma, metastatic lesion).

14. Renal mass biopsy or biopsy of metastatic lesion should be considered to obtain a diagnosis in patients with suspected metastatic renal cell carcinoma.

15. Renal mass biopsy should be performed prior to, or at the time of, thermal ablation or radiotherapy of the mass. 